\title{
Enseñanza de la estadística desde una perspectiva crítica
}

Autora: Lucía Zapata-Cardona

e-mail: luzapata@ayura.udea.edu.co

Dirección: Carrera 73 B N 75-191 (206)

Medellin, Colombia

Lugar de trabajo: Universidad de Antioquia,

Medellin, Colombia

Teléfono: (57) 3017181059

\section{Resumen}

El presente artículo presenta y discute una propuesta para la enseñanza de la estadística que se inspira en un paradigma sociocrítico de la educación matemática crítica. La propuesta se estructura a partir de investigaciones que buscan la formación del conocimiento estadístico mediante la resolución de problemas en contextos de la sociedad que puedan aportar a la ciudadanía crítica. El artículo también hace una revisión de literatura de diversas propuestas para la enseñanza de la estadística y discute los puntos comunes y las diferencias con las investigaciones al respecto. Al final se presentan tres ejemplos de investigaciones estadísticas que han sido desarrolladas en espacios escolares.

Palabras clave: enseñanza de la estadística, ciudadanía crítica, investigaciones estadísticas. Keywords: teaching statistics, critical citizenship, statistical investigations.

\section{Abstract}

This article presents and discusses a proposal for teaching statistics which is inspired in a socio-critical paradigm of critical mathematics education. The proposal is structured from statistical investigations seeking the formation of statistical knowledge thru problem solving in critical contexts of society that can support critical citizenship. The article also presents a literature review of different proposals for teaching statistics and discusses the points of agreement and differences with the statistical investigations. At the end, three examples of statistical investigations developed in the school system are presented. 


\section{Introducción}

Los estudiantes, en su paso por la educación obligatoria, aprenden una gran cantidad de información sobre conceptos y procedimientos estadísticos que son incapaces de utilizar cuando se enfrentan a problemas reales del mundo (Bakker, Van Mierlo y Akkerman, 2012). Aprenden a manipular símbolos estadísticos para desempeñarse bien en los exámenes escolares pero esas prácticas son inútiles en espacios fuera de la escuela (Roth, 1996). Este fenómeno revela una dicotomía entre el conocimiento escolar y el mundo de los estudiantes, la cual es promovida principalmente por la forma en que la estadística es enseñada - fragmentada y procedimental (algunos autores le llaman conocimiento inerte [Bakker y Derry, 2011]) y desconectada del contexto en el que el conocimiento es producido-. En el actual momento histórico - una era de la información y la tecnología- la escuela no necesita enfocarse en conocimiento factual sino en desarrollar habilidades de pensamiento que le permitan al estudiante entender su entorno y participar críticamente en sociedad.

A pesar del llamado a centrar la enseñanza de la estadística en procesos de investigación que incluyan producción de datos, medición y modelado de la variación, (Moore, 1997; Franklin et al., 2007), que responda a una visión holística de la estadística (Bakker, Van Mierlo, y Akkerman, 2012) y que refleje la práctica de los profesionales estadísticos (MacGillivray y Pereira-Mendoza, 2011), la enseñanza de la estadística sigue centrando su atención en el dominio de conceptos, procedimientos y representaciones gráficas. Así lo muestran el estudio de Zapata-Cardona (2014), en el cual las tareas de la clase de estadística se orientan primariamente a desarrollar procedimientos algoritmicos, y el estudio de Zapata-Cardona y Rocha (2016), en el cual las preguntas de los profesores privilegian respuestas exactas o aplicación de procedimientos. La escuela no debería centrarse en proveer a los estudiantes de información disciplinar aislada de su mundo sino formarlos para abordar críticamente las crisis y los conflictos de la sociedad. Es decir, formar a los estudiantes para que sean ciudadanos críticos. Así lo plantea Skovsmose inspirado en las ideas de Giroux: «la escuela debe educar a los estudiantes para ser ciudadanos críticos, preparados para correr riesgos, desafiar y creer que sus acciones pueden marcar una diferencia en la sociedad» (1999:26). Entendemos la ciudadanía crítica como una cualidad del pensamiento que ayuda a los ciudadanos a ser conscientes en cuanto a lo ambiental, social, político y económico y desarrolla disposiciones críticas hacia el mundo en el que viven (Stillman, Brown, Faragher, Geiger, y Galbraith, 2013).

Ese conocimiento estadístico que aprenden los estudiantes a lo largo de su formación escolar -inerte, fragmentado y desarticulado-, en el mejor de los casos, ayuda a desarrollar habilidades para aplicar procedimientos en problemas rutinarios tipo libro de texto. No obstante, ese conocimiento no logra transformar las realidades ambientales, sociales, políticas y económicas del mundo en el que están inmersos. La estadística es el resultado de una construcción humana, por ende, es una actividad que responde a los requerimientos de la cultura y que nace de las necesidades propias del ser humano. «Los objetos y la actividad científica son el resultado social y cultural» (Etchegaray, 2010:14), por lo tanto, la estadística no debería ser enseñada aislada de la condición humana, ni de los contextos sociales y culturales. En general, las ciencias son una respuesta a problemas de naturaleza antropológica que surgen con relación al entorno físico, biológico y social en el que vive el hombre (Etchegaray, 2010). Tomando en cuenta esta consideración, la enseñanza de la estadística debería atender a esa relación del ser humano con la naturaleza (en el sentido expresado por Radford, 2016).

La tradición en la enseñanza de la estadística ha privilegiado el uso de tareas en contextos hipotéticos con datos artificiales - no auténticos-que tienen muy poca relación con el mundo del estudiante y con fenómenos críticos de la sociedad. Así lo reveló un estudio que exploró las tareas de los libros de texto que se proponían a los estudiantes para la 
enseñanza de la estadística (Zapata-Cardona y Marrugo Escobar, 2016). Consideramos crucial organizar la enseñanza de la estadística a partir de contextos sociales críticos que permitan a los estudiantes desarrollar habilidades en el manejo de herramientas estadísticas pero también formar su consciencia social. En otras palabras, resaltar el poder formativo de la estadística y su condición de actividad humana.

En este artículo presentamos una propuesta para la enseñanza de la estadística que posibilita superar ese conocimiento estadístico inerte, fragmentado y desarticulado -desvinculado del mundo del estudiante y de los contextos sociales-y aportar a la comprensión y transformación de la sociedad. Es una propuesta integradora -inscrita en el paradigma sociocrítico-que vincula conceptos, razonamiento estadístico, procesos investigativos, contextos sociales reales -Skovsmose (1999) los llama «situaciones críticas»- para formar al ciudadano crítico.

\section{Las investigaciones estadísticas}

Las investigaciones estadísticas son una manera holística y práctica para organizar la enseñanza de la estadística, pero su principal objetivo es aportar al desarrollo de la ciudadanía crítica de los estudiantes. Hay autores inscritos dentro de la perspectiva crítica que tienen propuestas similares para la enseñanza de la estadística a las que llaman proyectos (ver, por ejemplo, Biajone, 2006; y Campos, 2016). Nosotros preferimos la expresión «investigaciones estadísticas» porque queremos resaltar ese proceso investigativo que tiene lugar cuando los estudiantes exploran y comprenden esos dilemas sociales e intentan reaccionar de manera crítica ante ellos. Las investigaciones estadísticas están inspiradas en la filosofía de la educación matemática crítica de Ole Skovsmose (1999) -quien sostiene que el objetivo de la enseñanza es el desarrollo de la ciudadanía crítica- pero también integra importantes desarrollos de la educación estadística, tales como: los procesos investigativos (Wild y Pfannkuch, 1999), la naturaleza de la práctica estadística (Batanero y Díaz, 2011a), los procesos de resolución de problemas (Franklin et al., 2007), la ambigüedad de los problemas reales (Makar y Fielding-Wells, 2011), el desarrollo del pensamiento estadístico (MacGillivray y Pereira-Mendoza, 2011; Wild y Pfannkuch, 1999), y la consideración de la variación en los fenómenos aleatorios (Franklin et al., 2007).

Las investigaciones estadísticas:

1) Incluyen todo un proceso de identificación de un problema o asunto de interés en un contexto particular. 2) Imitan la práctica diaria de los estadísticos profesionales que está centrada en la resolución de problemas reales (Pfannkuch y Wild, 2000; Wild y Pfannkuch, 1999) pero se enfocan en las situaciones críticas de la sociedad.

3) Conciben la estadística como un campo de conocimiento integrado que vincula conocimientos, procedimientos, habilidades y disposiciones para entender y participar críticamente en el mundo.

4) Vinculan el conocimiento producido en la vida diaria de los estudiantes -llamado por D'Ambrosio (1999) el «mundo de afuera»-con el conocimiento escolar. 5) Conciben el aprendizaje de la estadística y el desarrollo del pensamiento estadístico como procesos contextuales que se llevan a cabo dentro de experiencias de aprendizaje auténticas (MacGillivray y Pereira-Mendoza, 2011; Wild y Pfannkuch, 1999) encarnadas en conflictos y dilemas sociales. Cuestiones del mundo relacionadas con producción de basu$\mathrm{ra}$, indicadores de desarrollo humano, crecimiento demográfico, indices de desempleo, producción de alimentos, propagación de enfermedades, cambio climático, dieta, pobreza, represión, impacto ambiental tienen un buen potencial para contribuir a la conciencia social (Skovsmose, 1999; Stillman, Brown, Faragher, Geiger, y Galbraith, 2013).

6) No se centran exclusivamente en los saberes sino que toman en cuenta el desarrollo de disposiciones de pensamiento y la dimensión social de los seres (Radford, 2006). 
Puesto que el objetivo de las investigaciones estadísticas en el aula es el desarrollo de una ciudadanía crítica, a continuación discutimos este concepto. Según Skovsmose, «ser crítico significa prestarle atención a una situación crítica, identificarla, tratar de captarla, comprenderla y reaccionar frente a ella» (1999:16). «Ser crítico significa enfocarse en una situación crítica y buscar alternativas, tal vez reveladas por la situación misma. Significa tratar de identificar alternativas posibles» (18). Así, educar para la ciudadanía crítica se puede entender como estimular funciones del pensamiento que propicie que los estudiantes se conviertan en actores que comprendan, transformen su realidad -social, política, económica y ambiental-y contribuyan a la creación de condiciones más democráticas en la sociedad. La ciudadanía crítica lleva consigo una concepción particular de educación.

Si la educación pretende ser crítica, tiene que tener en cuenta el contexto crítico de la escolaridad y tratar de desarrollar posibilidades para crear una consciencia acerca de los conflictos, al igual que proporcionar las competencias que sean importantes para manejar tales situaciones críticas. (24-25)

Como consecuencia, los contextos en los que se inscriben las investigaciones estadísticas no pueden ser artificiales, hipotéticos o teóricos, sino contextos que vinculen aspectos críticos de la realidad social, política, económica y ambiental de los estudiantes.

La educación crítica es mucho más que únicamente enseñar a la gente iletrada las habilidades básicas. Naturalmente este es uno de los trabajos importantes de la educación, pero no puede identificarse como la (principal) característica de la educación crítica. La educación crítica debe luchar contra las restricciones ideológicas, debe reaccionar a los conflictos y a la diferenciación de oportunidades que la sociedad encarga a las escuelas y debe otorgar competencias que capaciten a la gente para confrontar la naturaleza crítica de la sociedad. (28)

\section{Otras propuestas para la enseñanza de la estadística}

Muchos autores han sugerido propuestas para la enseñanza de la estadística que a primera vista podrían parecer similares a las investigaciones estadísticas. Sin embargo, hay claras diferencias especialmente en términos de los objetivos, del contexto en el que se inscriben y de la concepción de sujeto que llevan consigo. Aquí se discuten algunas de ellas.

Por ejemplo, Makary Fielding-Wells (2011) describen las investigaciones estadísticas como problemas pobremente estructurados y ambiguos que se usan como una herramienta pedagógica para que la indagación empírica - como la propuesta por Wild y Pfannkuch (1999) - tenga lugar en el aula de clase. Para Makar y Fielding-Wells, la característica principal de lo que llaman investigaciones estadísticas es la pobre estructura de los problemas. Las autoras argumentan que usualmente los problemas que se proponen a los estudiantes en la estadística escolar son muy bien estructurados, la planeación, la recolección de datos, el análisis y las conclusiones no son problemáticos y las decisiones más cruciales con relación al proceso investigativo quedan ocultas a sus ojos. Sin embargo, el trabajo del profesional estadístico está lleno de ambigüedades que deben ser tratadas y direccionadas. Los problemas pobremente estructurados requieren un profundo debate para negociar las características que se medirán para dar solución al problema bajo investigación. Algunos de los ejemplos que Makar y Fielding-Wells (2011) plantean como investigaciones estadísticas son: «¿Cuál marca de goma de mascar es la mejor?» «¿Cuál es el mejor diseño para un avión de papel?». Pero, si bien estas investigaciones estadísticas coinciden con nuestra propuesta en algunos aspectos - promover la indagación empírica a partir de problemas abiertos que generen un ciclo de indagación empírica-, los contextos dentro de los que se desarrollan no tienen un propósito social. El contexto, para las autoras, es un conjunto de referencias que la situación evoca. Así, el objetivo de la propuesta de Makar y 
Fielding-Wells difiere del objetivo de la nuestra. En contraste, MacGillivray y Pereira-Mendoza (2011) proponen la enseñanza de la estadística a partir de procesos investigativos - de problemas reales en contextos reales - a los que llaman «proyectos estadísticos». Para estos autores los

proyectos estadísticos bien sean grandes o pequeños, ofrecen aprendizaje experiencial de las investigaciones estadísticas. Tal aprendizaje vincula conceptos, conocimiento y habilidades en contextos que pueden atraer y motivar los estudiantes, como también enseñarles sobre los diferentes matices del pensamiento estadístico, las fluctuaciones de los datos y los desafios de comunicar e interpretar en contexto. (112)

Una característica importante de los proyectos estadísticos es que son investigaciones de libre elección puesto que nacen de los intereses de los estudiantes y son ellos quienes deciden el tópico de estudio, exploran y analizan sus datos y producen un reporte escrito. Esta característica garantiza que los estudiantes se apropien de su proyecto. Estos proyectos:

capturan los desafios de transformarideas y preguntas en planes de investigación, los aspectos prácticos y el desorden de la recolección y manipulación de datos, lo esencial de la elección y uso de herramientas estadísticas, y la sintesis de interpretaciones estadísticas en contextos reales y auténticos. (113)

En los proyectos estadísticos el contexto es muy importante. La mayoría de ellos se gesta en contextos reales y da origen a la producción de datos reales. Sin embargo, la naturaleza de los contextos reales es diferente de la que nosotros proponemos en las investigaciones estadísticas. En estos proyectos, el contexto es real pero no se centran necesariamente en fenómenos de naturaleza social. Por ejemplo, algunos de los propuestos por MacGillivray y Pereira-Mendoza (2011) son: ¿Cómo se investiga la elasticidad de los gusanos de golosina? ¿Cómo aplaude la gente? ¿La forma de aplaudir tiene relación con la forma en que las personas cruzan sus brazos? Desde ese punto de vista, los proyectos estadísticos son herramientas potentes para promover el pensamiento estadístico y la estadística como una herramienta de indagación, pero se quedan cortos para promover la conciencia social respaldada en las investigaciones estadísticas.

Batanero y Díaz también sugieren la enseñanza de la estadística con proyectos y argumentan que estos «están concebidos para introducir en la clase una filosofía exploratoria y participativa» (2011a:46) y que son herramientas útiles para promover el razonamiento y la cultura estadística. Para estas autoras, los proyectos son investigaciones realistas y abiertas en las que se propone un problema práctico y se usa la estadística para resolverlo. Pueden ser sugeridos por el profesor o escogidos libremente por los estudiantes y avanzan por un ciclo investigativo: planteamiento de un problema, decisión sobre los datos a recoger, recolección y análisis de datos y obtención de conclusiones sobre el problema planteado.

Una de las justificaciones que dan las autoras para trabajar con proyectos es que la estadística es inseparable de las aplicaciones. Mediante el trabajo con proyectos la estadística se concibe como una herramienta en la toma de decisiones y en la investigación o trabajo profesional. Los conceptos y técnicas estadísticas se introducen siempre en el contexto de una investigación. Son las preguntas estadísticas las que motivan la introducción de conceptos y cualquier tipo de herramienta.

Algunos ejemplos de estadística con proyectos son: Comprueba tus intuiciones sobre el azar (Bataneroy Arteaga, 2011). ¿Cómo son los alumnos de tu clase? (Batanero y Díaz, 2011b). Las matemáticas de la catadora de café (Batanero, 2011). Supervivencia en el Titanic (Díaz, Cañadas y Batanero, 2011).

Es claro que en esta propuesta los contextos son cruciales para conectar la estadística con las aplicaciones reales. Esos contextos están fundamentados en fenómenos aleatorios que pueden ser simulados, o en datos demográficos recogidos en la clase me- 
diante encuestas, o en datos históricos. No obstante, funcionan como punto de partida para el desarrollo de funciones mentales del sujeto y en el desarrollo de ciertos contenidos estadísticos. Por esta razón. creemos que el sujeto al que se dirige la propuesta estadística con proyectos es un sujeto epistémico -en el sentido descrito por Radford, 2011-, quien dará significado al conocimiento, mientras que nuestra propuesta investigaciones estadísticas se refiere a un sujeto social -concreto, contextual, cultural-que busca despertar en los estudiantes la responsabilidad por fenómenos de naturaleza social. La Guía para la Evaluación e Instrucción en Educación Estadística (GAISE por sus siglas en inglés [Franklin et al., 2007]) también propone un marco teórico a partir de la resolución de problemas estadísticos como un proceso investigativo que involucra cuatro componentes: formular preguntas, recolectar datos, analizar datos, interpretar resultados. En este marco teórico el contexto es un elemento importante para la resolución de problemas estadísticos, pues el mismo provee el significado a los datos, «los datos no son solo números sino números con un contexto» (Franklin et al.:7). Desde este punto de vista, el contexto es un dispositivo para conectar la estadística con las aplicaciones prácticas - «"aquello que acompaña” al aprendizaje cuando los estudiantes desarrollan una actividad», según Valero (2002:50) - y ayuda a la comprensión conceptual y procedimental de la estadística, no obstante la manera en que se concibe el contexto es insuficiente para generar consciencia sobre asuntos de naturaleza social. Algunos de los problemas estadísticos que propone la GAISE son: ¿Usan los libros de quinto grado palabras más largas que los libros de tercer grado? ¿Es la estatura un predictor útil de la extensión de brazos para los estudiantes de nuestra escuela? ¿Cómo el nivel de luz solar afecta el crecimiento de una planta? ¿Qué tipo de música es más popular entre los estudiantes de nuestra escuela?

Santos y Ponte (2013) proponen el desarrollo de investigaciones estadísticas en la clase como una estrategia de trabajo interdisciplinario para promo- ver el desarrollo del conocimiento estadístico, las habilidades de comunicación y la argumentación en los estudiantes. Dicha propuesta considera que quienes emprenden investigaciones estadísticas progresan por etapas -como las sugeridas por Wild y Pfannkuch, 1999- que incluyen el planteamiento de preguntas estadísticas, diseño de planes de recolección de información, análisis, interpretación y crítica de los datos, y argumentos e inferencias. Los contextos en los que estos autores inscriben las investigaciones estadísticas son bastante diversos e incluyen temáticas variadas como: deportes, gastronomía, consumo de agua, juegos, pasatiempos, alimentación entre otros.

Pero el objetivo de las investigaciones estadísticas sugeridas por Santos y Ponte (2013) se centra en el desarrollo de funciones cognitivas del estudiante. Resaltamos que, aunque en nuestra propuesta también hay un profundo interés por el desarrollo de un conocimiento estadístico, nuestro objetivo va mucho más allá. Las investigaciones estadísticas que proponemos buscan que el estudiante ponga todo ese conocimiento disciplinary las habilidades adquiridas en los procesos investigativos al servicio de la formación de su consciencia social y de la transformación de la sociedad.

\section{Algunos ejemplos de investigaciones estadísticas}

Las siguientes experiencias se han llevado a cabo en la escuela obligatoria en diferentes niveles educativos. Aunque se aborda una temática estadística -0 varias simultáneamente-a partir de una investigación estadística, el estudio detallado de la situación crítica lleva a los estudiantes a que reaccionen ante la misma y propongan alternativas de solución. De esta manera la investigación estadística no termina cuando el estudiante domina los procedimientos y herramientas estadísticas sino cuando los usa para aborda las crisis de la sociedad. 


\subsection{Ciberdependencia}

Una profesora identificó la dependencia a los dispositivos móviles que usaban sus estudiantes. Para orientar el estudio del problema e intentar una posible solución propuso a sus estudiantes de décimo grado la siguiente pregunta estadística: ¿Quiénes son más ciberdependientes, los hombres o las mujeres de la institución? Los estudiantes diseñaron instrumentos, recogieron información y la analizaron hasta llegar a contraste de hipótesis y finalizaron haciendo propuestas de intervención en la institución educativa. Se resalta de esta experiencia la lista de estrategias que los estudiantes plantearon para ayudar desde la institución a reducir la ciberdependencia. Esta experiencia está publicada en Zapata-Cardona, González, y Ceballos (2015).

\subsection{Equidad de género}

Se inicia con la lectura y discusión de la noticia llamada «Pasajeros se bajan del avión al enterarse de que las pilotos eran mujeres» (La Opinión, 2016). A continuación, los estudiantes buscan los datos de las 10 actrices $y 10$ actores mejor pagados durante el 2015 (ver Figura 1). Con los datos recolectados, los estudiantes hacen un estudio exploratorio hasta llegar a contrastes de hipótesis. Seguidamente, se lleva a cabo la experiencia Evidencia estadística de discriminación (Scheaffer, Gnanadesikan, Watkins y Witmer, 1996), en la cual 48 gerentes de banco estudian la misma hoja de vida (24 marcadas como hombre y 24 marcadas como mujer) para recomendar su ascenso (21 hombres y 14 mujeres se recomiendan para ascenso). Los estudiantes exploran a partir de distribuciones teóricas y de simulación (con manipulativos y con software) si hay evidencia estadística de discriminación. A continuación, se estudian los salarios de hombres y mujeres en Colombia. Finalmente, los estudiantes discuten ideas para reducir acciones discriminatorias entre hombres y mujeres en diferentes espacios: la familia, la escuela, torneos deportivos, el barrio, etcétera.

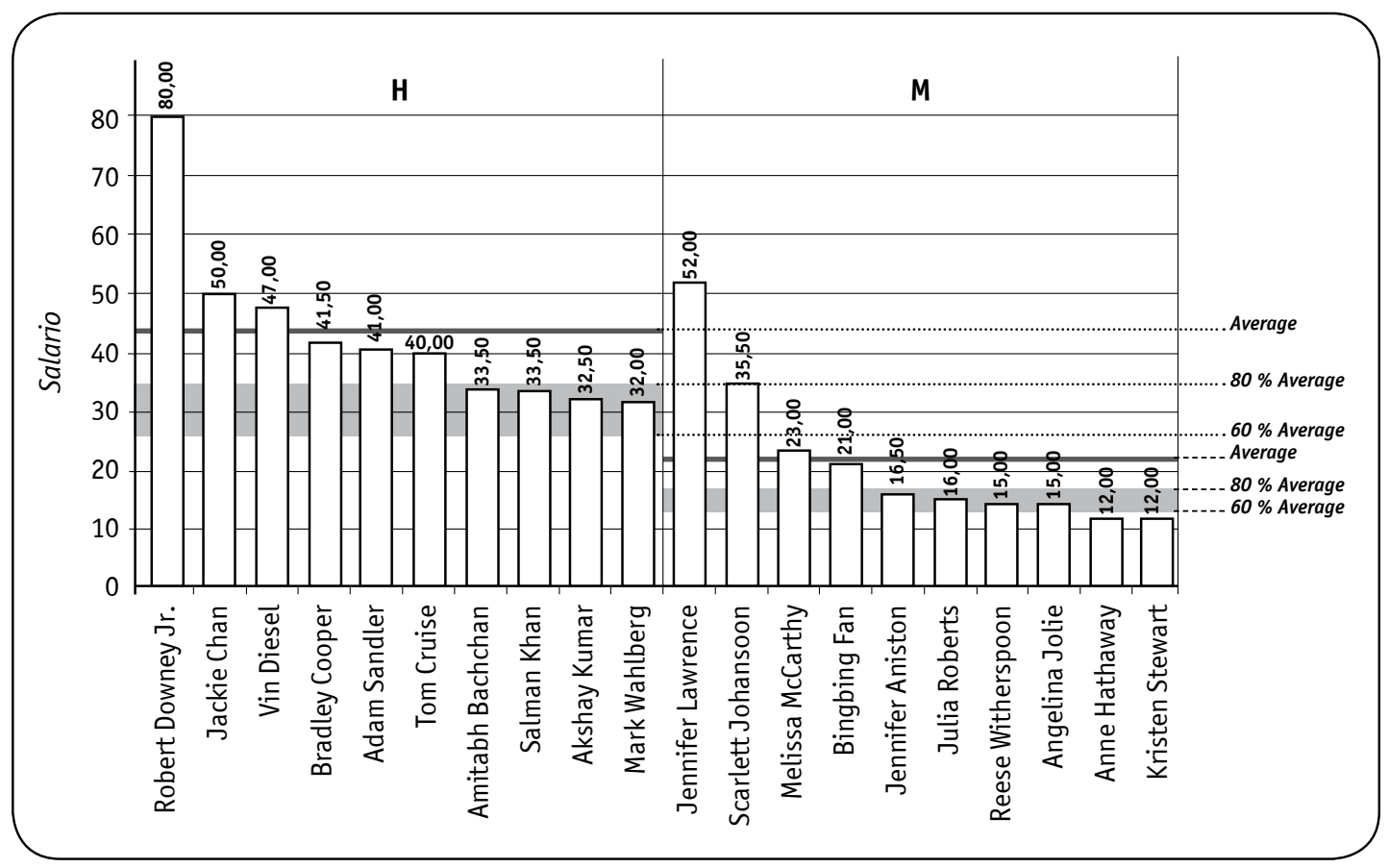

Figura 1.

Salarios de actores y actrices mejor pagados durante 2015 en millones de dólares. 


\subsection{Obesidad infantil}

Después de leer la noticia «La obesidad infantil es una epidemia mundial: OMS» (El Espectador, 2016), los estudiantes de quinto grado se preguntan cómo la institución educativa contribuye a esta crisis social. Deciden estudiar la pregunta estadística: ¿Cuál es el valor nutricional de los productos de nuestra tienda escolar? Hacen una lista de los productos que vende la cafetería escolar y consultan la información nutricional de cada uno (cantidad en gramos, calorías por porción, gramos de grasas, gramos de carbohidratos, gramos de proteínas, miligramos de sodio, y si es o no alimento procesado). Analizan los datos usando exclusivamente representaciones gráficas y descubren, entre otros aspectos, que la tienda escolar tiene productos con alto nivel calórico -o sódico- pero con pobre valor nutricional. Sugieren al personal de la cafetería reemplazar aquellos productos identificados como «nutricionalmente pobres» por otros de alto valor nutricional.

\section{Implementación de las investigaciones estadísticas}

Siendo coherentes con una mirada crítica de la enseñanza de la estadística, sería contradictorio hablar de formas preestablecidas para implementar las investigaciones estadísticas en el aula de clase. También sería contradictorio hablar de una estimación uniforme en el tiempo requerido para su implementación. Como se indicó previamente, cada investigación estadística parte de una situación crítica particular que tiene su propia naturaleza, sus propias características y sus propias tensiones. No obstante, las investigaciones estadísticas se inician con el estudio detallado de la situación crítica en cuestión para que los estudiantes puedan comprender el escenario de partida. En ocasiones es necesario generar datos (en el ejemplo Equidad de género la simulación con manipulativos, en el ejemplo Ciberdependencia la aplicación de la encuesta) o tomar datos de fuentes oficiales (en el ejemplo Equidad de género los salarios entre hombres y mujeres de Colombia). Dependiendo del escenario se pueden usar manipulativos o software para estudiar empíricamente los datos recolectados. Los conceptos y herramientas estadísticas surgen de acuerdo a la necesidad del escenario y se afilan con cada uso. Algunas veces se pueden requerir herramientas sofisticadas como el contraste de hipótesis (ejemplos: Ciberdependencia y Equidad de género) y en otras las exploraciones gráficas podrían ser suficientes (ejemplo:Obesidad infantil). Una investigación estadística concluye cuando el estudiante ha comprendido y explorado empíricamente la situación crítica y es capaz de usar su juicio para establecer conclusiones prácticas bien sea para atender el fenómeno o para transformar su estado actual (propuestas de intervención para reducir la ciber-dependencia, reconocer y sancionar acciones discriminatorias, reemplazar productos nutricionalmente pobres por otros de alto valor nutricional). En otras palabras, cuando hay evidencia que el estudiante no solo se apropió de un concepto o procedimiento estadístico, sino que encontró una solución a un dilema social y aportó elementos para transformar la situación crítica o para reaccionar frente a ella, podemos decir quetiene lugar el proceso de formación de la conciencia social. En ese momento se puede hablar de aprendizaje como un fenómeno social, «aprender no es simplemente apropiarse de algo 0 asimilar algo, sino que es el proceso mismo en que se forman nuestras capacidades humanas» (Radford, 2006:114). Elaprendizaje es una toma de consciencia (Skovsmose, 1999) - consciencia no como un elemento metafísico sino como esa relación con el mundo concreto-y discernimiento crítico (Radford, 2014).

Una de las grandes ventajas de las investigaciones estadísticas es que pueden ser introducidas en cualquier nivel de la educación obligatoria (incluyendo prescolar) y pueden extenderse incluso hasta la formación universitaria. Además, son tan versátiles, que una sola investigación estadística puede dinamizar el estudio de múltiples conceptos (en el ejemplo Equidad de género se trabaja: contraste de hipótesis, error tipo Iy tipo II, asociación de variables, dependencia e independencia estadística, nivel de significancia, distribuciones muestrales). 


\section{Conclusiones}

Aunque muchos autores hayan planteado propuestas similares a las investigaciones estadísticas en el aula de clase, nuestra propuesta se caracteriza por conectar el conocimiento escolar con los contextos críticos del mundo de los estudiantes. Las investigaciones estadísticas, además de promover el pensamiento estadístico, los procesos de investigación y la resolución de problemas, la estadística como un conocimiento holístico inseparable de las aplicaciones, busca que los estudiantes participen activamente de la sociedad en la que viven. Las investigaciones estadísticas a partir de situaciones críticas del mundo son insumos importantes para promover la consciencia social.

Las diferentes propuestas para la enseñanza de la estadística que se presentaron en la revisión de literatura llevan consigo una comprensión de saber, de sujeto y de contexto que difieren de nuestra propuesta. Por ejemplo, para algunas de esas propuestas, el saber está asociado con el dominio de contenidos estadísticos y del desarrollo de funciones mentales del sujeto. Mientras que para nosotros el saber es un proceso de naturaleza social: «una forma cultural e históricamente constituida de reflexión y acción incrustada en prácticas sociales» (Radford, 2011:43). Desde nuestra propuesta de investigaciones estadísticas, la producción del saber va más allá del desarrollo del pensamiento estadístico. La producción del saber está mediado por formas culturales que sostienen la comprensión y la transformación del mundo de las personas y los conceptos que ellas se forman del mundo (Radford, 2011).

En términos de la comprensión de sujeto también hay grandes diferencias. Para muchas de las propuestas para la enseñanza de la estadística presentadas, el aprendiz es un sujeto epistémico quien con sus funciones mentales transforma el conocimiento y le da sentido. Mientras que en nuestra propuesta de investigaciones estadísticas el aprendiz es un sujeto social que participa de una cultura.

La concepción del contexto también se comprende de forma diferente. Para algunas de las propuestas para la enseñanza de la estadística, el contexto se asocia con ese escenario que acompaña el aprendizaje de los estudiantes, puede referirse a las nociones y procedimientos dentro de los cuales se ubica el problema o a las referencias que evoca en el estudiante. No obstante, esos contextos no logran desarrollar la conciencia sobre asuntos de naturaleza social que apuntan al desarrollo de una ciudadanía crítica. Los contextos a los que nosotros nos referimos en las investigaciones estadísticas aportan a la ciudadanía crítica y deben estar asociados con situaciones críticas de la sociedad que el estudiante pueda estudiar, comprender y transformar críticamente.

Las personas con una buena formación estadística no se vuelven por si mismos ciudadanos críticos.

Suponer que la enseñanza de las matemáticas [estadística] de por sí contribuye a la formación de ciudadanos democráticos porque ofrece un conocimiento que es indispensable y necesario en las sociedades modernas equivale a suponer que el conocimiento matemático [estadístico] en sí es poderoso y deseable. (Valero, 2002:53)

Las investigaciones estadísticas se convierten en un instrumento para conectar ese conocimiento estadístico con el mundo. Es decir, responden a esa relación del ser humano con la naturaleza. También son una propuesta inspirada en la matemática crítica que busca que los estudiantes usen la estadística para encontrar solución a los dilemas sociales. Asimismo, promueven el aprendizaje de la estadística como un cuerpo holístico que articula conocimiento estadístico, habilidades de pensamiento, y procesos investigativos pero su fin último es la formación de la consciencia ciudadana para entender y participar críticamente en el mundo. Además, son una estrategia que conecta el saber escolar con los contextos ambientales, sociales, políticos y económicos en los que está inmerso el estudiante armonizando esa relación del ser humano con la naturaleza. 
Agradecimientos: este trabajo fue apoyado por el Comité de Investigación de la Universidad de Antioquia -CODI- en su Convocatoria Programática Ciencias Sociales, Humanidades y Artes 2012. Agradecemos a los árbitros anónimos quienes con sus pertinentes y valiosos comentarios contribuyeron a mejorar este escrito.

\section{Referencias bibliográficas}

Bakker, A., \& Derry, J. (2011). Lessons from Inferentialism for Statistics Education [Lecciones del inferencialismo para la educación estadística]. Mathematical Thinking and Learning, 13(1-2), 5-26. doi: 10.1080/10986065.2011.538293

Bakker, A., Van Mierlo, X. \& Akkerman, S. (2012). Learning to integrate statistical and work related reasoning [Aprendiendo a integrar razonamiento estadístico con razonamiento laboral]. 12th International Congress on Mathematical Education. Seoul, Korea.

Batanero, C. (2011). Las matemáticas de la catadora de té. En C. Batanero, \& C. Díaz, La estadística con proyectos (pp. 149-174). Granada: Universidad de Granada.

Batanero, C. \& Arteaga, P. (2011). Comprueba tus intuiciones sobre el azar. En C. Batanero \& C. Díaz, La estadística con proyectos (pp. 47-71). Granada: Universidad de Granada.

Batanero, C. \& Díaz, C. (2011a). La estadística con proyectos. Granada: Universidad de Granada.

(2011b). ¿Cómo son los alumnos de tu clase? En C. Batanero \& C. Díaz, La estadística con proyectos (pp. 73-95). Granada: Universidad de Granada.

Biajone, J. (2006). Trabalho de Projetos: possibilidades e desafios na formação formação estatística do Pedagogo [Trabajo de proyectos: posibilidades y desafios en la formación estadística del pedagogo]. Campinas, SP: Tesis de maestría no publicada. Facultad de Educación Universidad Estatal de Campinas - UNICAMP.

Campos, C.R. (2016). La educación estadística y la educación crítica. Segundo Encuentro Colombiano de Educación Estocástica (2 ECEE). Bogotá, Colombia.

D'Ambrosio, U. (1999). Literacy, matheracy and technocracy: a trivium for today [Literacia, materacia y tecnocracia]. Mathematical thinking and learning, 1(2), 131-153.

Díaz, C., Cañadas, G. \& Batanero, C. (2011). Supervivencia en el Titanic. En C. Batanero \& C. Díaz, La estadística con proyectos (pp. 219-246). Granada: Universidad de Granada.

Etchegaray, S.C. (2010). Reflexiones y aportes para ayudar a re-pensar la enseñanza de las matemáticas. Yupana, 5, 11-23. doi:www.dx.doi.org/10.14409/yu.v1i5.258

Franklin, C., Kader, G., Mewborn, D., Moreno, J., Peck, R. \& Perry, M. (2007). Guidelines for assessment and instruction in statistics education (GAISE) report: A pre-K-12 curriculum framework [Guia para la evaluación e instrucción en educación estadística]. Alexandria, VA: American Statistical Association.

MacGillivray, H., \& Pereira-Mendoza, L. (2011). Teaching statistical thinking through investigative projects [Enseñando pensamiento estadístico meediante proyectos investigativos]. En C. Batanero, G. Burrill, \& C. Reading, Teaching statistics in school mathematics-Challenges for teaching and teacher education: A joint ICMI/IASE Study (págs. 109-120). Springer Science+Business Media. doi:10.1007/978-94-007-1131-0_14

Makar, K., \& Fielding-Wells, J. (2011). Teaching teachers to teach statistical investigations [Enseñando a los profesores a enseñar investigaciones estadísticas]. En C. Batanero, G. Burrill, C. Reading, \& A. Rossman, Teaching statistics in school mathematics - Challenges for teaching and teacher education: a joint ICMI/IASE study: the 18th ICMI study (págs. 347-358). Dordrecht: Springer. doi:10.1007/978-94-007-1131-0_33

Moore, D. (1997). New pedagogy and new content: The case of statistics [Nueva pedagogía y nuevo contenido: el caso de la estadísitca]. International Statistical Review, 65(2), 123-137. 
Pfannkuch, M. \& Wild, C.J. (2000). Statistical thinking and statistical practice: Themes gleaned from professional statisticians [Pensamiento estadístico y práctica estadística]. Statistical Science, 15(2), 132-152.

Ponte, J.P. (2011). Preparing teachers to meet the challenges of statistics education [Preparando profesores para asumir los desafios de la educación estadística] . En C. Batanero, G. Burrill, C. Reading \& A. Rossman, Teaching statistics in school mathematics - Challenges for teaching and teacher education: a joint ICMI/IASE study: the 18th ICMI study (pp. 299-309). Dordrecht: Springer. doi:10.1007/978-94-007-1131_29

Radford, L. (2006). Elementos de una teoría cultural de la objetivación. Relime, Número Especial, 103-129.

- - (2011). La evolución de paradigmas y perspectivas en la investigación. El caso de la didáctica de las matemáticas. En J. Vallès, D. Álvarez \& R. Rickenmann, L'ctivitat docent intervenció, innovació, investigació (pp. 33-49). Girona, España: Documenta Universitaria.

- - (2014). De la teoría de la objetivación. Revista Latinoamericana de Etnomatemática, 7(2), $132-150$.

(2016). On alienation in the mathematics classroom [Sobre la alineación en la clase de matemáticas]. International Journal of Educational Research, 79, 258-266. doi:http://dx.doi. org/10.1016/j.ijer.2016.04.001

Roth, W.-M. (1996). Where is the context in contextual word problems? Mathematical practices and products in Grade 8 students' answers to story problems [¿Dónde está el contexto en problemas contextuales? Cognition and Instruction, 14, 487-527.

Santos, R. \& Ponte, J.P. (2013). 0 desenvolvimento de investigações estatísticas: Um estudo com futuros professores e educadores [El desarrollo de investigaciones estadísticas: Un estudio con profesores y futuros profesores]. En J.M. Contreras, G.R. Cañadas, M.M. Gea \& P. Arteaga, Actas de las Jornadas Virtuales en Didáctica de la Estadística Probabilidad y Combinatoria (pp. 455-462). Granada: departamento de Didáctica Universidad de Granada. Obtenido de file:///C:/Users/Facultad\%20Educacion/Downloads/Dialnet-0DesenvolvimentoDeInvestigacoesEstatisticas-4770378.pdf

Scheaffer, R., Gnanadesikan, M., Watkins, A. \& Witmer, J. (1996). Activity-based Statistics [Estadística basada en actividades]. New York: Springer-Verlag.

Skovsmose, 0. (1999). Hacia una filosofía de la educación matemática crítica. (P. Valero, Trad.) Bogotá: Una Empresa Docente (Trabajo original publicado en 1994).

Stillman, G., Brown, J., Faragher, R., Geiger, V. \& Galbraith, P. (2013). The role of textbooks in developing a socio-critical perspective on mathematical modeling in secondary classrooms [El rol de los libros de texto en el desarrollo de una perspectiva socio-crítica en la modelación matemática en los salones de secundaria]. En G.A. Stillman, Teaching mathematical modelling: Connection to research and practice. International perspectives on the teaching and learning of mathematical modelling (pp. 361-371). Dordrecht: Springer Science + Bussiness. doi:10.1007/978-94-007-6540-5_30

Valero, P. (2002). Consideraciones sobre el contexto y la educación matemática para la democracia. Quadrante, 11(1), 33-40.

Wild, C. \& Pfannkuch, M. (1999). Statistical thinking in empirical enquiry (with discussion) [Pensamiento estadístico en la indagación empírica (con discusión)]. International Statistical Review, 67(3), 223-265.

Zapata-Cardona, L. (2014). Alcance de las tareas propuestas por los profesores de estadística. Uni-pluri/versidad, 14(1), 53-62.

Zapata-Cardona, L. \& Marrugo Escobar, L. (2016). Critical citizenship in colombian statistics textbook [Ciudadanía crítica en los libros de texto de estadística colombianos]. Proceedings 13th International Congress on Mathematical Education (ICME 13). Hamburg, Germany. 
Zapata-Cardona, L. \& Rocha, P. (2016). Teachers' questions in the statistics class [Preguntas de los profesores en la clase de estadística]. En D. Ben-Zvi \& K. Makar, The teaching and learning of statistics: International perspectives (pp. 271-278). Springer International Publishing. doi:10.1007/978-3319-23470-0_32

Zapata-Cardona, L., González, D. \& Ceballos, Z. (2015). Colaboración entre profesores de estadística e investigadores: Una experiencia de aula. Revista Colombiana de Matemática Educativa, 1(1), 602-607.

\section{Fuentes periodísticas}

El Espectador (25 de enero de 2016). La obesidad infantil es una epidemia mundial: OMS. Obtenido de http://www.elespectador.com/noticias/salud/obesidad-infantil-una-epidemia-mundial-omsarticulo-612592

La Opinión (17 de julio de 2016). Pasajeros se bajan del avión al enterarse de que las pilotos eran mujeres. Obtenido de http://www.laopinion.com/2016/07/14/pasajeros-se-bajan-del-avion-alenterarse-de-que-las-pilotos-eran-mujeres/ 